\section{Klimaschutz „Made in China“}

\author{
Um die in Paris vereinbarten globalen Klimaschutzziele zu errei- \\ chen, müssen vor allem die größten Emittenten von Treibhaus- \\ gasen einen relevanten Beitrag leisten. Dass China mit der landes- \\ weiten Einführung eines $\mathrm{CO}_{2}$-Emissionshandels einen großen \\ Schritt in diese Richtung geht, ist ein Hoffnungsschimmer am \\ sonst eher düsteren klimapolitischen Himmel. \\ Von Achim Lerch, Sven Rudolph und Joseph Dellatte
}

\begin{abstract}
hina ist mit einem Anteil von mehr als $25 \%$ der weltweit größte $\mathrm{CO}_{2}$-Emittent absolut und wirtschaftet nach wie vor mit einer Kohlenstoffintensität, die deutlich über dem globalen Durchschnitt liegt. Die Erreichung der Klimaziele von Paris ist daher ohne immense Klimaschutzanstrengungen im Reich der Mitte nicht denkbar. Umso erfreulicher, dass China nun mit der Einführung eines landesweiten $\mathrm{CO}_{2}$-Emissionshandelssystems einen großen Schritt in diese Richtung unternimmt.

Chinas Klimapolitik wird dabei insbesondere von der Nationalen Entwicklungs- und Reformkommission gesteuert, der weitere Behörden in den Provinzen unterstehen und die durch einige Forschungsinstitutionen unterstützt werden, etwa dem Energy Research Institute (ERI) oder dem beim Finanzministerium angesiedelten China Clean Development Fund Managing Center.
\end{abstract}

\section{Pilotphase in ausgewählten Regionen}

Bereits 2011 startete in China der Emissionshandel in sieben Pilotregionen, womit bereits $18 \%$ der chinesischen Bevölkerung und 20\% der Emissionen erfasst wurden. Ende $2016 \mathrm{kam}$ noch eine weitere Provinz hinzu. Da es bislang keine nationale Regulierung gab, war jedes Handelssystem anders organisiert und reguliert, was entsprechend zu sehr unterschiedlichen Ergebnissen führte so schwankten etwa die durchschnittlichen Preise pro Tonne $\mathrm{CO}_{2}$ zwischen
3,7 Yuan (Chongqin) und 36,5 Yuan (Peking) (1 Yuan [CNY] = 0,13 EUR, Stand: 1. 12. 2017).

Gemeinsam ist allen Pilotregionen ein Emissionshandelssystem, das aus fünf Elementen besteht: Einem System der Zertifikateallokation (Cap-andTrade), einem System der Bewertung und Erfüllung, einem Handelssystem, einem System für Monitoring, Reporting and Verification sowie einem Registrierungssystem. Insgesamt funktioniert der Emissionshandel nach Ansicht des ERI noch nicht zufriedenstellend, unter anderem ist die Allokation der Zertifikate noch nicht effizient und es muss das Bewusstsein für die Notwendigkeit eines Emissionsmanagements in den Unternehmen geschärft werden.

\section{Verknüpfung mit Europa}

Insbesondere die chinesische Regierung drängt auf eine landesweite Ausweitung des Emissionshandels, die Forschungsinstitute sind diesbezüglich eher skeptisch. Nachdem zumindest bereits erste Schritte zur Vereinheitlichung der regionalen Systeme erfolgt sind, sollen weitere Angleichungen der Regulierungen sowie eine Verknüpfung der acht Pilotsysteme erfolgen. Ein funktionierendes landesweites Handelssystem soll entsprechend dem Fünfjahresplan bis spätestens 2020 etabliert sein.

Von besonderem Interesse sowohl für die globale Klimapolitik wie auch für den europäischen Emissionshandel ist die erklärte Absicht Chinas, sein Emis- sionshandelssystem mit dem europäischen bis $2025 \mathrm{zu}$ verknüpfen. Betrachtet man zudem die bereits bestehenden beziehungsweise in Entstehung befindlichen Emissionshandelssysteme in anderen Teilen der Welt und auf allen Governance-Ebenen, vor allem in Nordamerika (Lerch et al. 2017 beziehungsweise Rudolph et al. 2017), aber zum Beispiel auch in Neuseeland oder auf lokaler Eben in Tokyo (Rudolph et al. 2016), steigen somit die Chancen auf die Etablierung eines globalen Kohlenstoffmarktes, wie er dem globalen Umweltproblem Klimawandel angemessen wäre, erheblich. Je größer der Markt und damit auch die Unterschiede in den Grenzvermeidungskosten, umso besser kann das Instrument des Emissionshandels seine Vorteile ausspielen und die Kosten der weltweiten Klimapolitik insgesamt erheblich senken, was für deren Erfolg ein entscheidender, wenn nicht sogar der entscheidende Faktor sein dürfte (Lerch 2015). Man darf also gespannt sein, ob China nun auch in der Klimapolitik vom Nachahmer zur Führungsmacht wird.

\section{Literatur}

Lerch, A. (2015): Emissionshandel reloaded. In: ÖkologischesWirtschaften 30/4: 12.

Lerch, A./Rudolph, S./Kawakatsu, T. (2017): Emissionshandel von unten. In: Zeitschrift für Umweltpolitik und Umweltrecht 40/2: 113-133. Rudolph, S./Kawakatsu, T./Morotomi, T./Lerch, A. (2017): Für eine föderale marktbasierte Klimapolitik. In: ÖkologischesWirtschaften, 32/1: 41-45.

Rudolph, S./Morotomi, T. (2016): Acting Local! An Evaluation of the First Compliance Period of Tokyo's Carbon Market. In: Carbon and Climate Law Review 10/1: 75-78.

AUTOREN + KONTAKT

Dr. Achim Lerch ist Professor für Volkswirtschafts lehre an der FOM Hochschule in Kassel.

FOM Hochschule, Kölnische Straße 69 34117 Kassel. Tel.: +49 $20181004-0$ E-Mail: achim.lerch@fom.de

Dr. Sven Rudolph ist Associate Professor und Joseph Dellatte ist Masterstudent an der Graduate School of Economics, Kyoto University.

Kyoto University, Yoshida-Honmachi, Sakyu-Ku, Kyoto. Tel.: +8175 7533400, 